\title{
TECHNICAL REPORTS
}

\section{Engineering microRNA responsiveness to decrease virus pathogenicity}

\author{
Elizabeth J Kelly, Elizabeth M Hadac, Suzanne Greiner \& Stephen J Russell
}

The cellular tropisms of eukaryotic viruses are shaped by their need for entry receptors and intracellular transcription factors. Here we show that viral tropisms can also be regulated by tissue-specific microRNAs (miRNAs). Target sequences complementary to muscle-specific miRNAs were inserted into the $3^{\prime}$ untranslated region (UTR) of an oncolytic picornavirus that causes lethal myositis in tumor-bearing mice. The recombinant virus still propagated in subcutaneous tumors, causing total regression and sustained viremia, but could not replicate in cells expressing complementary miRNAs and therefore did not cause myositis. This altered tropism was not due to insertional attenuation, as a control virus containing a 3' UTR insert with a disrupted miRNA target sequence fully retained its lethal myotropism. Tissue-specific destabilization of viral genomes by miRNA target insertion provides a potentially versatile new mechanism for controlling the tropism of replicating viruses for therapy and may serve as a new modality for attenuating viruses for vaccine purposes.

MiRNAs are $\sim 20-24$-nucleotide regulatory RNAs anticipated to modulate the expression of genes involved in nearly all cellular activities, in addition to having roles in development ${ }^{1}$, cancer ${ }^{2}$ and disease susceptibility ${ }^{3}$. MiRNA distribution analysis has revealed that each cell contains a diverse population of miRNAs and that the abundance of individual miRNA species varies greatly between different cell lineages and different tissues ${ }^{4}$. MiRNAs have recently been shown to modulate the translation and degradation of sequencecomplementary cellular messenger RNAs, thereby playing a major part in the tissue-specific regulation of gene expression patterns ${ }^{5}$. Sequence complementarity in the seven-nucleotide 'seed sequence' is essential for suppression of mRNA translation, and catalytic mRNA degradation can be seen upon high sequence complementarity between the miRNA and its target (miRT) ${ }^{5}$.

Oncolytic viruses, used experimentally as cancer therapeutics for over 100 years $^{6}$, are increasingly transitioning into clinical trials ${ }^{7}$. Methods to engineer specificity and improve safety by means of transcriptional and transductional targeting have been ongoing for many years ${ }^{8}$. However, new targeting modalities are needed for next-generation oncolytic viruses (particularly those not amenable to previous strategies), gene therapy vectors and the attenuation of new viral pathogens for therapeutic or vaccine purposes.

MiRNAs have previously been exploited to regulate nonreplicating lentiviral vectors through a method in which gene expression of integrated transgenes is controlled by either cellular ${ }^{9}$ or viral ${ }^{10}$ miRNAs. The modification of viral replication cycles, however, has not previously been accomplished by this means. Modifying the host range of a replication-competent virus via miRNA target insertion has great appeal, in that it should be highly economical in terms of sequence insert size as compared to other targeting strategies. RNA viruses that have historically proven difficult to target might be particularly vulnerable to this means of restriction, as their genomes should be susceptible to miRNA-mediated attack immediately upon entering the target cell cytoplasm.

In this study, we modulated the tropism of a well studied picornavirus by engineering target sequences for known cellular miRNAs into its genome.

\section{RESULTS}

CVA21 is a potent oncolytic that causes severe myositis

Coxsackievirus A21 (CVA21) is a pathogenic picornavirus known to cause respiratory tract infections and myositis in human subjects ${ }^{11,12}$. It causes rapid-onset lethal myositis both in suckling mice ${ }^{13}$ and in immunocompromised mice bearing subcutaneous human myeloma xenografts (Fig. 1). The virus is cytolytic in a variety of cancer cell lines (Fig. 1a $)^{14}$. CVA21 is potently oncolytic in tumor-bearing mice (Fig. 1b,c $)^{15}$, mediating rapid regression of large tumors over a short time span, which is followed by severe, progressive muscle paralysis necessitating euthanasia within $12 \mathrm{~d}$ of virus exposure (Fig. 1d). This phenotype is observed even at very low doses $(1 \times$ $10^{3}$ half-maximal tissue culture infective dose $\left(\mathrm{TCID}_{50}\right.$; data not shown)). To determine the cause of this paralysis, we harvested biopsies from mice with paralysis and performed tissue overlays on H1-HeLa cells to look for viral recovery (Supplementary Table 1 online). Virus was present only in residual tumor tissue and skeletal muscle and was absent, most notably, from central nervous system tissue (Supplementary Table 1). Upon histological examination, we found that CVA21-treated mice had massive inflammation and necrosis in skeletal muscle, indicative of a severe myositis, whereas 

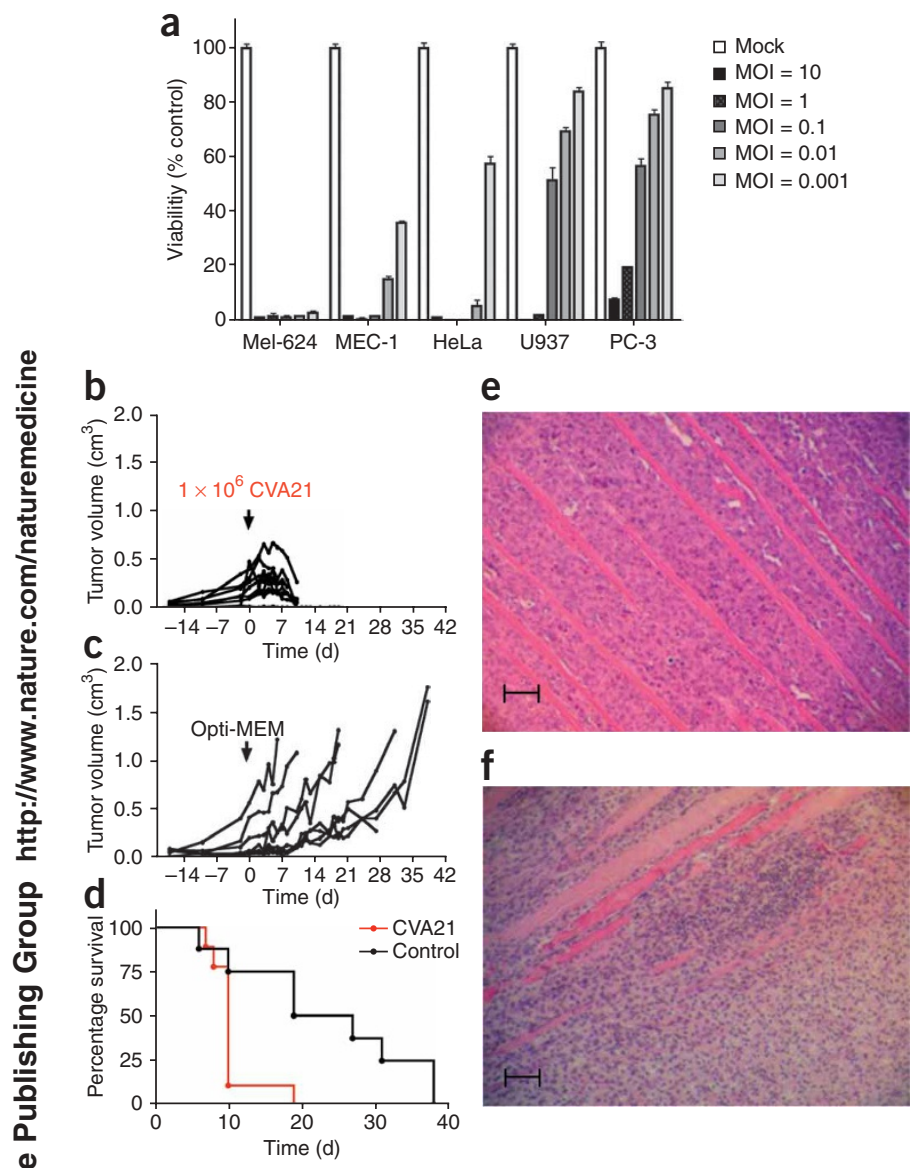

control Opti-MEM-treated mice had no such histological findings (Fig. 1e,f). Of note, myositis occurred only in tumor-bearing mice, indicating that the tumors probably provided a reservoir of virus amplification that resulted in massive viremia, allowing viral spread to muscle tissue.

Given that CVA21 can destroy both tumor and muscle tissue in immunocompromised mice, it presented us with an opportunity to explore the question of whether miRNAs could potentially recognize and selectively regulate RNA stability such that they could modulate
Figure 1 Efficient oncolysis by CVA21 is associated with severe and fatal myositis. (a) Susceptibility of cancer cell lines to the cytolytic effects of CVA21. Error bars represent means \pm s.d. $(\mathbf{b}, \mathbf{c})$ Tumor volumes of SCID mice carrying subcutaneous Kas 6/1 human myeloma xenografts were treated with $1 \times 10^{6}$ WT CVA21 TCID 50 (b) or an Opti-MEM control (c). (d) KaplanMeier survival curves of Opti-MEM control-treated $(n=8)$ and CVA21treated $(n=9)$ mice. (e,f) Histological skeletal muscle sections isolated from muscle adjacent to rear femurs of control (e) and CVA21-treated (f) mice. Scale bar, $0.1 \mathrm{~mm}$.

viral host range. By engineering target elements into the viral genome for three previously identified muscle-specific miRNAs highly conserved between rodents and humans (miR-1, miR-133a, miR-206) ${ }^{16}$, we investigated the ability of CVA21 therapy to control the fatal myositis without perturbing its potent oncolytic activity.

\section{Muscle-specific miRNAs downregulate gene expression}

To select the optimal target configuration for the destabilization of viral mRNA, we used a previously described nonreplicating lentiviral reporter system ${ }^{9}$. To maximize the probability of catalytic transcript degradation, we chose target sequences bearing perfect sequence complementarity to cellular miRNAs and inserted multiple copies of each sequence into the vector genome ${ }^{17}$.

Four copies of control hematopoeitic cell-specific miR-142 3pT, muscle-specific miR-133T, muscle-specific miR-206T, or two copies each of miR-133T and miR-206T were inserted downstream of EGFP in an HIV-based lentiviral vector, and vector-driven gene expression was monitored in a panel of cell lines, including HeLa cells, which do not express cognate miRNA to the inserted targets $^{18}$ (Fig. 2a,b). Targets for miR-133T or miR-206T caused EGFP expression to be selectively downregulated in TE-671 and L6 muscle cells, and vectors with two copies of each of these muscle-specific targets were able to silence more efficiently (Fig. 2c). When TE-671 or L6 cells were cultured in horse serum, which induces the cells to differentiate into myotube cells expressing higher levels of muscle-specific miRNAs ${ }^{19}$, EGFP expression was suppressed even more (Fig. 2d). As expected, vectors carrying the hematopoetic cell-specific miR-142 3p target were selectively downregulated in hematopoetic Kas 6/1 cells (Fig. 2d).

\section{MiRNAs are capable of regulating CVA21 life cycle}

Having identified the best target sequences for downregulation of gene expression in muscle cells, we constructed an miRNA-regulated

Figure 2 Analysis of lentiviral vectors incorporating muscle-specific miRNA targets in muscle and nonmuscle cells. (a) Schematic diagram of lentiviral vector containing miRNA targets. LTR, long terminal repeat; GA, Gag; SFFV, spleen focus-forming virus (promoter); $\psi$, packaging signal; RRE, Rev response element; CPPT, central polypurine tract. (b) Sequence of inserted target elements hematopoetic-specific miR-142 3pT (purple), muscle-specific miR133T (red), muscle-specific miR-206T (blue) and combined miR-133T and miR-206T, with spacer elements in black. (c) GFP positivity in cells transduced at an $\mathrm{MOI}$ of 1.0 with lentiviral vectors containing miR-142-3pT, miR-133T, miR-206T or both miR-133T and miR-206T. $* * P<0.001$ for Kas $6 / 1$ cells compared to HeLa cells. (d) GFP positivity in cells transduced as in c but in the presence of differentiation medium containing horse serum. ${ }^{*} P<0.01$ for TE671 and $\mathrm{L} 6$ cells compared to HeLa cells. Error bars represent means \pm s.d.; $n=3$.
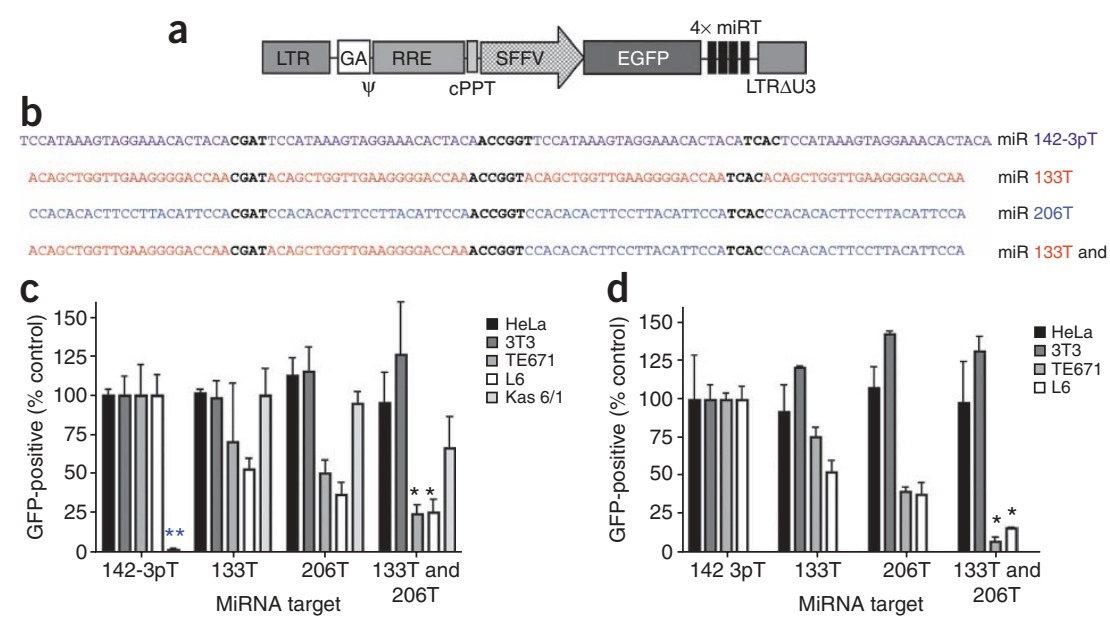


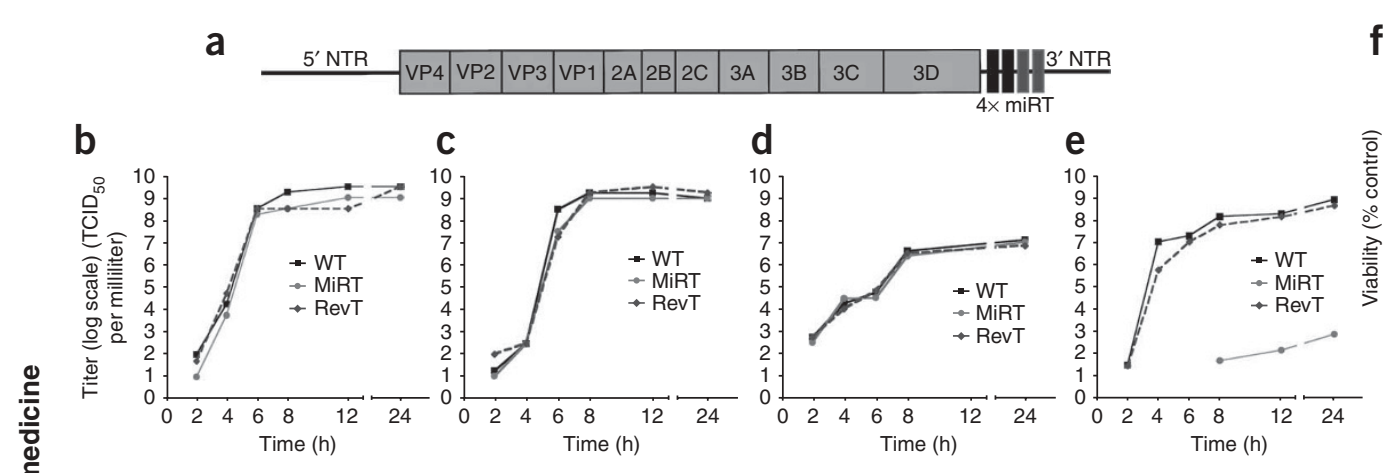

Figure 3 Construction and characterization of recombinant CVA21. (a) Schematic diagram of miRNA-targeted CVA21.

(b-e) One-step growth curves of WT, miRT and RevT CVA21 viruses in H1-HeLa cells (b), Mel 624 cells (c), Kas 6/1 cells (d) and H1-HeLa cells with miR-133 and miR-206 mimics (e). (f) Viability $24 \mathrm{~h}$ after viral infection at an MOI of 1.0 with WT, miRT or RevT CVA21 pretreated for $4 \mathrm{~h}$ with $200 \mathrm{nM}$ miRNA mimics (labeled on $x$ axis). $n=4$; ${ }^{*} P<0.01$ for miRT virustransfected cells compared to cells transfected with miRNA control. (g) Viral titers in supernatants collected $24 \mathrm{~h}$ after infection from cells infected with WT, miRT- or RevT CVA21 in the presence of miRNA mimics (representative of five repeat experiments). (h) In vitro survival of non-muscle (H1-HeLa) or muscle (TE671) cells when transfected with $1 \mu \mathrm{g}$ WT or miRT CVA21 RNA in 24-well plates, as determined by MTT assay. $n=3$; ${ }^{*} P<0.01$ for TE-671 cells compared to $\mathrm{H} 1-\mathrm{HeLa}$ cells. Error bars represent means \pm s.d.
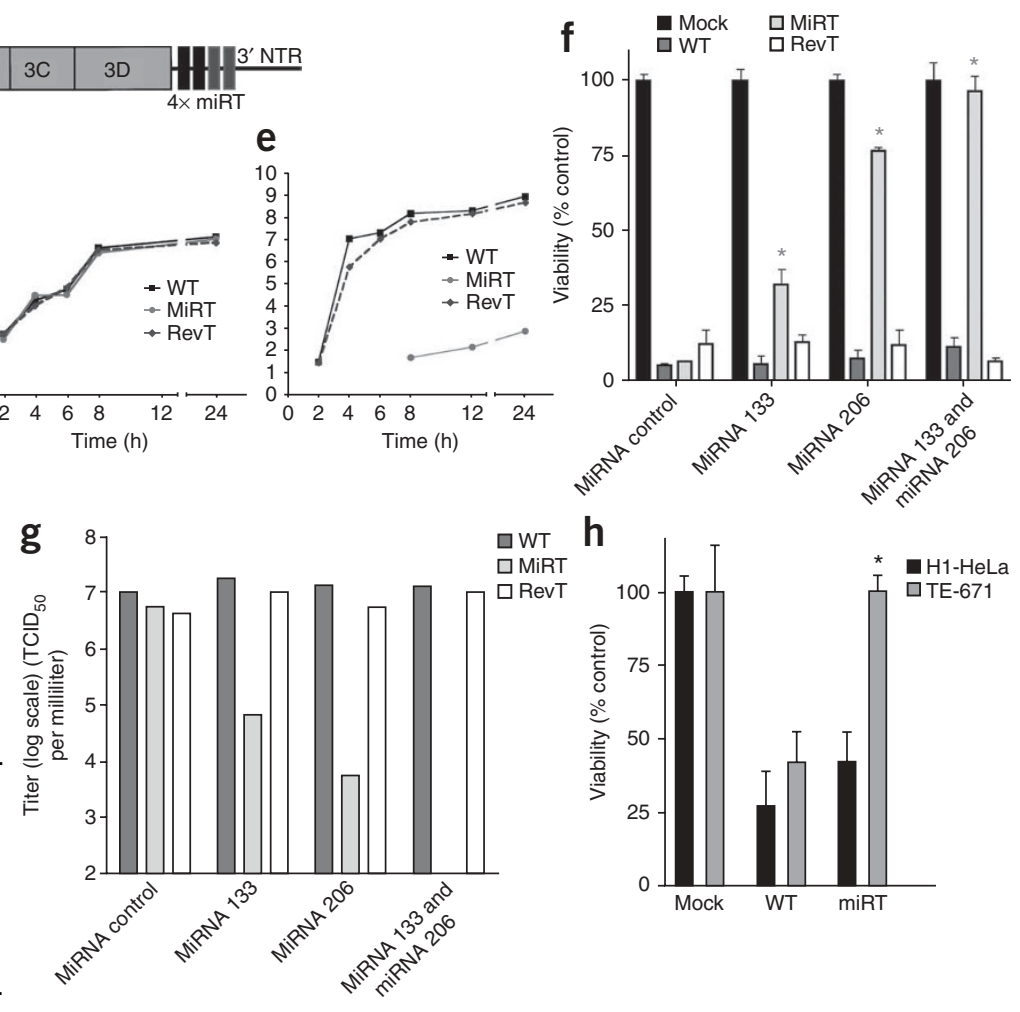

CVA21. Two copies each of miR-133T and miR-206T were inserted in the $3^{\prime}$ UTR. Previous reports have indicated that the $3^{\prime}$ UTR of picornaviruses is tolerant of sequence insertion ${ }^{20}$, and, therefore, miRTs were inserted at this site (Fig. 3a).

We next recovered infectious miRT virus (CVA21 with two copies each $133 \mathrm{~T}$ and $206 \mathrm{~T}$ in the $3^{\prime}$ UTR) by RNA transfection in H1-HeLa cells and compared its replication kinetics with those of the parental wild-type (WT) strain CVA21. The growth kinetics of these two viruses were indistinguishable in H1-HeLa, Mel-624 melanoma cells and Kas 6/1 multiple myeloma cells (Fig. 3b-d) and did not differ from the growth of a control virus (RevT, described below) carrying a control insert in the $3^{\prime}$ UTR.

To determine whether the lytic effects of the recombinant miRT CVA21 could be controlled by muscle-specific miRNAs, we infected CVA21-susceptible H1-HeLa cells with test and control viruses (at a multiplicity of infection (MOI) of 1.0) after transfection with synthetic dsRNA corresponding to human miRNAs (miRNA mimics) corresponding to miR-133T or miR-206T (Fig. 3e) or with a control mimic corresponding to a Caenorhabditis elegans miRNA that has no identified target in mammalian cells. The mimics of miR-133T or miR-206T each partially protected the H1-HeLa cells from viral lysis by -CVA21, with miR-206T providing greater protection than miR133T (Fig. 3f). When cells were exposed simultaneously to both of the muscle-specific miRNA mimics, they appeared to be fully resistant to the retargeted virus, such that cell viability was not significantly different from that of mock-infected cells $(P=0.49$; Fig. 3f). To determine whether propagation of miRT CVA21 was efficiently blocked by the muscle-specific miRNAs in a sequence-specific manner, we also measured the supernatant virus titers in this experiment. The virus titers in cells infected with miRT CVA21 were substantially decreased by miR-133 (two-log reduction) or miR-206 (three-log reduction) when the mimics were applied individually but were decreased to undetectable levels (more than five-log reduction) in the presence of both muscle-specific mimics (Fig. 3g). Moreover, in CVA21 genome transfer studies, endogenously expressed miRNAs in TE671 muscle cells afforded selective protection from the cytopathic effects of miRT CVA21 (Fig. 3h) $(P<0.01)$.

miRT CVA21 retains oncolytic activity and protects from myositis To investigate whether miRT CVA21 retains oncolytic efficacy in vivo and provides a protection phenotype against fatal myositis, we treated immunodeficient mice carrying subcutaneous human myeloma or melanoma xenografts (Fig. 4a-c, Supplementary Fig. 1a-d and Supplementary Fig. 2a-c online). Mice carrying established subcutaneous tumors were treated with a single intratumoral dose of $1 \times 10^{6}$ TCID $_{50}$ of each virus and monitored for tumor growth and survival (Fig. 4). WT CVA21-treated mice had rapid tumor regression, but all developed hind-limb paralysis and were killed in fewer than $15 \mathrm{~d}$. However, mice treated with the miRT virus had slow but eventually complete tumor regression and significantly increased survival as compared to WT CVA21-treated mice $(P<0.001$; Fig. 4d). Histological analysis of muscle tissue in mice treated with WT virus again showed massive infiltration and necrosis (Supplementary Fig. 3c,d online), whereas mice treated with miRT virus were rescued from this phenotype (only one mouse had pathologic evidence of myositis, and it was determined later that the miRT insert was mutated) (Supplementary Fig. 3e,f). Although survival was highly statistically significant (Fig. 4e; $P<0.001$ versus control and WT CVA21), a small number of mice developed tremors and labored breathing and, in two cases, paralysis and were euthanized. Pathologic examination of these mice indicated that these symptoms were the result of a polio-like myelitis rather than myositis (Fig. 5a,b). We next analyzed serum collected from all mice at two-week intervals after CVA21 treatment. Mice treated with miRT CVA21 had a lower but persistent viremia, as compared to mice treated with WT CVA21 (Fig. 4f), that may have allowed a retrograde axonal transport of the virus to 

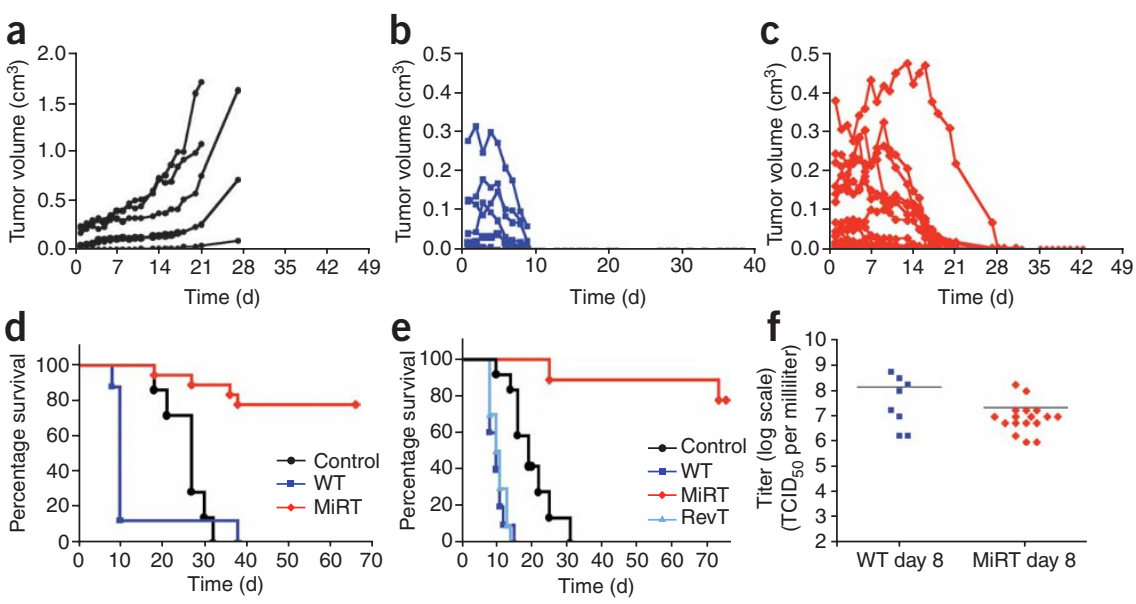

Figure 4 MiRNA-targeted CVA21 retains in vivo oncolytic activity and prolongs survival. (a-c) SCID mice carrying subcutaneous multiple myeloma xenografts were treated with an Opti-MEM control $(n=6 ; \mathbf{a}), 1 \times 10^{6}$ WT CVA21 $(n=8 ; \mathbf{b})$ or $1 \times 10^{6}$ miRT CVA21 $(n=18$; c). (d) Kaplan-Meier survival graphs of the mice in a-c. (e) Kaplan-Meier survival graphs of mice treated with one intratumoral dose of $1 \times 10^{6}$ WT $(n=9)$, miRT $(n=9)$ or RevT $(n=10)$ CVA21. (f) Viral titers collected from mice treated with WT or miRT-CVA21. Horizontal lines represent means. seen in WT CVA21-treated mice, which persisted in some mice, enabling us to analyze the stability of the miRT insert. Although RNA interference against viruses that infect vertebrates is not thought to occur naturally via miRNA targets encoded within viral genomes, we show that regulation by the miRNAprimed RNA interference machinery can be achieved by incorporation of (or the engineering of) tissue-specific miRNA targets into the genome sequence of the oncolytic virus. To determine whether viruses evolve to avoid miRTs within their viral genomes, we examined the stability of the insertions $45 \mathrm{~d}$ after virus administration (Fig. 5c). We reasoned that by this time the input virus would replicate extensively and, in light of the high error rate of RNA-dependent RNA polymerases ${ }^{22}$, would have ample opportunity to mutate the inserted sequence. Six of eleven viremic mice maintained viruses with (or near) 100\% sequence identity with the original sequence; three had viruses with $>80 \%$ sequence homology with the inserted miRTs; one had occur, as previously reported in intercellular adhesion molecule1-transgenic mice ${ }^{21}$.

To determine whether the altered in vivo host range properties of the miRT virus might be a nonspecific consequence of placing a 100-base pair insert into its $3^{\prime}$ UTR, we characterized the RevT virus (so called because of the revertant phenotype it showed in mice), which differs from miRT CVA21 only in the insert sequence. RevT CVA21 retains a full-length $3^{\prime}$ UTR insert with only minimal homology to the original miRNA target sequence (Supplementary Fig. $4 \mathbf{b}$ online). When cloned into the lentiviral EGFP reporter vector, the RevT insert was unable to mediate muscle cell-specific gene silencing (Supplementary Fig. 4c). Furthermore, the in vivo behavior of the RevT virus was indistinguishable from that of the WT virus (Supplementary Figs. 1 and 2). RevT-challenged mice died within $14 \mathrm{~d}$ of virus administration from severe, generalized myositis.

To address the issue of viral escape from engineered restriction of replication in muscle, we analyzed serum collected from all mice at two-week intervals after CVA21 treatment. Mice treated with miRT CVA21 initially had high level viremia, consistent with the viremia a virus that retained only $68 \%$ of the inserted target and one had a virus with limited sequence retention (the RevT virus). All mice maintained perfect homology to the WT virus in the $3 \mathrm{D}^{\mathrm{pol}}$ virus gene and 3' UTR sequence flanking the insert. Of the mice that had mutated the miRNA target insert, mice 3 and 11 lived free of symptoms to the terminal point of $70 \mathrm{~d}$ in the study. Mouse 5 never lost limb function but began to lose weight at day 60 after injection.

Because we observed sequence alterations within the miRNA target inserts that could decrease the efficiency with which the endogenous muscle-specific miRNAs recognized miRT CVA21, we analyzed the amount of creatine kinase secreted into the serum as a quantitative marker for muscle damage. WT CVA21-treated mice had creatine kinase levels up to 22-fold higher than did control mice, whereas creatine kinase abundance in miRT virus-treated mice did not significantly differ from the control mice at day 45 after treatment (Fig. 5d). At the terminal point of $70 \mathrm{~d}$, however, we observed a slight increase in secreted creatine kinase in the serum in both mice carrying mutations within miRT inserts (mice 5 and 11), and the average
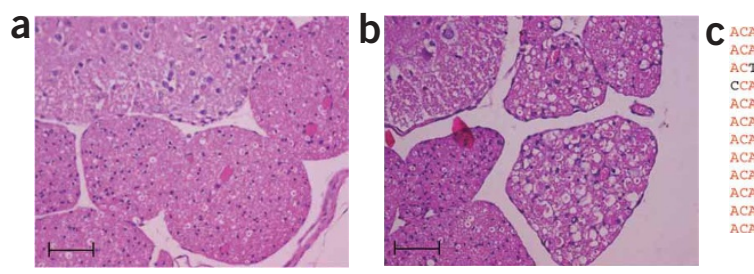

Figure 5 Characterization of miRNA-targeted CVA21 in vivo. (a) Posterior spinal cord section of a nonsymptomatic miRT virus-treated mouse. (b) H\&E-stained posterior spinal cord section of an miRTtreated mouse presenting with tremors, labored breathing and hind limb paralysis. (c) Sequence alignment of the 3 'UTR inserts from viruses collected from the sera of mice 1-11 on day 45 after treatment. (d) Creatine kinase concentration in mouse serum from control, WT CVA21-infected or miRT CVA21-infected mice. The closed box indicates mouse 5 . The dashed box refers to mouse 11 . Scale bars, $0.1 \mathrm{~mm}$.
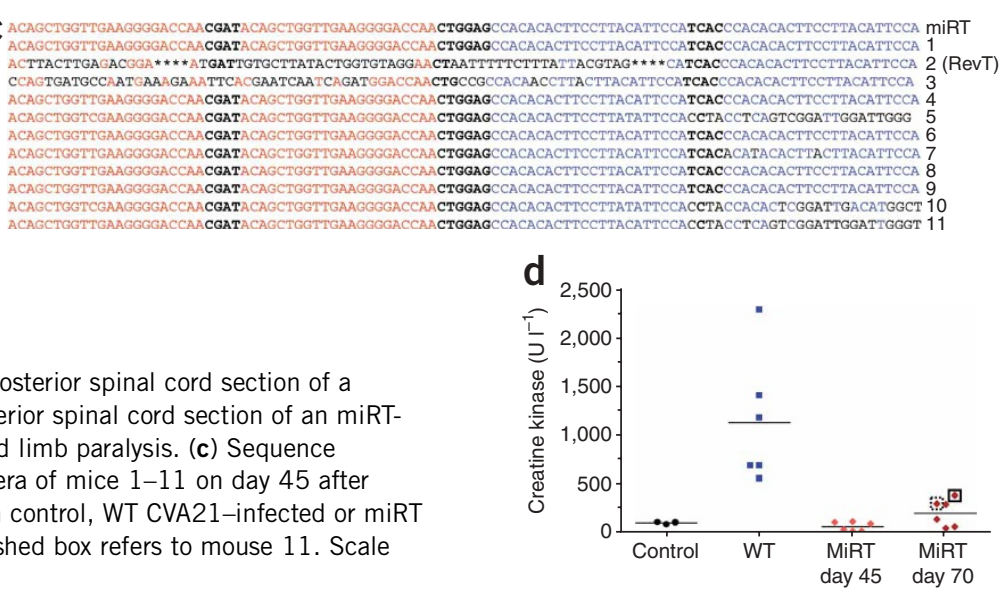
increase in creatine kinase abundance in these mice was statistically significant as compared to control mice ( $P=0.0088$; Fig. 5d).

\section{DISCUSSION}

Oncolytic viruses are currently being engineered such that they are more potent and interact synergistically with chemotherapy ${ }^{23}$, radiotherapy ${ }^{24}$ and immunotherapy ${ }^{25}$. As their potency increases, toxicity may follow. Thus there is a need for more stringently regulated virus tropism. Unlike transductional targeting strategies that may actually e increase the pathogenicity of viruses by increasing their host range, miRNA-mediated regulation is highly unlikely to do so, as it restricts host range by using pre-existing cellular miRNAs to act as a primed antiviral defense mechanism. Many of the most potently active virotherapeutics both emerging and antiquated are associated with well documented toxicities to the brain (for example, poliovirus, herpes simplex virus, vesicular stomatis virus, Egypt 101 virus and West Nile virus) ${ }^{6,26}$, heart (for example, reovirus) ${ }^{27}$ or liver (for example, adenoviruses) ${ }^{28}$. Incorporation of tissue-specific miRNA targets into these viruses might increase their therapeutic index without perturbing their antitumor activity.

Previous reports have indicated that four tandem-repeat copies of a single target element are most efficacious for the silencing of gene expression ${ }^{17}$. Here we show that this efficacy may be target dependent, as we found that combining two tandem repeats of two musclespecific miRNA target elements has an additive effect in restricting expression of a transgene in cells expressing cognate miRNAs. We have data that support the hypothesis that this effect is related to the concentrations of the relevant miRNAs in the target cell cytoplasm (Supplementary Fig. 5 online): in the presence of very high concentrations of a single miRNA, the synergistic effect is almost entirely lost.

In addition to the applications for virotherapy, miRNA-mediated regulation may prove, in time, to have its greatest impact on vaccine development. With the continued inability of existing vaccines to prove efficacious for eradicating poliomyelitis (owing to reversion of the vaccine strain $)^{29}$, modifications to include neuron-specific miRNA targets may prove highly beneficial. MiRNA-mediated restriction provides a unique method for making a pathogen selectively impotent in replicating in its tissue of choice and provides a new paradigm for generating de novo vaccines and improving the safety of existing vaccines.

The efficacy of miRNA-mediated targeting depends in part on the ability of the virus to escape from cellular miRNAs, as has been seen with exogenously introduced small interfering RNAs against viruses ${ }^{30}$. Sequence analysis in our system has shown that although viruses can escape, this happened only in a minority of the mice treated with virus. In addition, the spread of viruses used as oncolytics is often hampered by an immune response against the virus such that viremia is quickly cleared ${ }^{31}$. Because antibodies against the virus would probably be produced before a viral escape mutant arose in the case of both virotherapy and vaccines, viral escape is less likely to be a handicap than with small interfering RNA antiviral therapy. However, because it is unlikely that a virus could be engineered such that it would be unable to mutate target inserts, the best way to avoid escape mutants is probably increasing the number of target elements and insertion sites.

MiRNAs are emerging as new potent and active regulators of cellular gene expression. Here we show that naturally occurring and differentially expressed miRNAs can also be exploited to modulate viral replication cycles. Overall, our data provide proof of principle that the life cycles of replication-competent viruses can be modified by way of host-encoded miRNAs to direct tropism and enhance safety of virotherapeutics and provide a new paradigm for the attenuation of pathogens and the creation of safer vaccines.

\section{METHODS}

Cell culture, transfections and lentiviral vector production. We obtained H1-HeLa, L6, TE-671, C2C12, 293T and 3T3 cells from American Type Culture Collection and maintained them in DMEM supplemented with 10\% FBS (also referred to as growth medium) in 5\% $\mathrm{CO}_{2}$. Kas 6/1 multiple myeloma cells were kindly provided by D. Jelenik and were grown in RPMI Medium supplemented with 10\% FBS and interleukin-6. Mel-624 melanoma cells were provided by R. Vile and were grown in DMEM supplemented with $10 \%$ FBS. We differentiated cells in DMEM supplemented with $2 \%$ horse serum for $4 \mathrm{~d}$. We performed transfections with the Promega Calcium Phosphate ProFection Mammalian Transfection System with a total of $3 \mu \mathrm{g}$ of DNA per well in a six-well plate. Briefly, we transfected cells $24 \mathrm{~h}$ after they had been plated in $2 \mathrm{ml}$ of medium at $2.5 \times 10^{5}$ cells per well. We then collected the cells or used them for immunofluorescence $72 \mathrm{~h}$ after transfection. We obtained lentiviral vectors by transfection of $10 \mu \mathrm{g}$ of each lentiviral transfer plasmid (pHR-sin-CSGW dlNot1 or pHR-sin-F.Luc, generously provided by Y. Ikeda) and lentiviral packaging plasmid (cytomegalovirus $\Delta \mathrm{R} 8.91$ ) and $3 \mu \mathrm{g}$ of the vesicular stomatitis virus $\mathrm{G}$ protein packaging construct pMD.G in a T-75 flask. We collected the supernatants $72 \mathrm{~h}$ after transfection and filtered them through a $0.45-\mu \mathrm{m}$ syringe filter.

Plasmid construction. We obtained miRNA sequences from the Sanger Institute miRBase. We designed oligonucleotides using a previously described method $^{9}$ and annealed them in equimolar amounts in STE buffer (10 mM Tris$\mathrm{HCl}, 0.1 \mathrm{M} \mathrm{NaCl}$ and $1 \mathrm{mM}$ EDTA) by heating the solution to $94{ }^{\circ} \mathrm{C}$ followed by gradual cooling at $23{ }^{\circ} \mathrm{C}$. We cloned the annealed oligonucleotides into the XhoI-NotI site of pHR-sin-CSGW dlNotl and produced the lentiviral vectors as described above.

Flow cytometry. We plated $2.5 \times 10^{5}$ cells in six-well plates with DMEM containing $10 \%$ FBS and infected them with HIV-luciferase vectors. We collected the cells $72 \mathrm{~h}$ after transfection for flow cytometry. We fixed the cells for flow cytometry in $4 \%$ paraformaldehyde in PBS, washed them and resuspended in PBS with 2\% FBS. We quantified GFP fluorescence with the Becton Dickinson FACScan flow cytometer. We analyzed FACS data with BD CellQuest Software.

Recombinant CVA21 construction. The pGEM-CVA21 clone was kindly provided by M. Gromeier. We cloned miRTs into the $3^{\prime}$ UTR of pGEMCVA21 in between base pairs 7,344 and 7,345 by overlap extension PCR.

Virus and viral RNA production. We produced viral RNA with the Ambion Megascript and Megaclear T7 polymerase kit according to the manufacturer's instructions. We transfected $1 \mu \mathrm{g}$ RNA per well into H1-HeLa cells seeded in 12-well plates with the Mirus RNA transfection reagent, and at $24 \mathrm{~h}$ we scraped the wells and collected cell pellets. We subjected the cell pellets to three freezethaw cycles in liquid nitrogen, cleared the cell debris by centrifugation and added the cleared lysate to H1-HeLa cells in a T-75 flask. For miRT CVA21, three passages were necessary to obtain suitable titers of virus.

CVA21 titration. We performed titration of CVA21 on H1-HeLa cells. We plated the cells in 96-well plates at 50\% confluence. After $24 \mathrm{~h}$, we made serial tenfold dilutions $\left(1 \times 10^{-2}\right.$ to $\left.1 \times 10^{-10}\right)$ of the virus and added $100 \mu \mathrm{l}$ of each dilution to each of eight duplicate wells. After incubation at $37{ }^{\circ} \mathrm{C}$ for $72 \mathrm{~h}$, we fixed and stained the wells with a solution of $0.1 \%$ crystal violet, $20 \%$ methanol and $4 \%$ paraformaldehyde. We then assessed the wells for cytopathic effects, which manifests as nonstaining areas devoid of viable cells. If we observed purple-staining cells on $75 \%$ or less of the well surface, then we scored the well as positive. We determined TCID $_{50}$ values with the Spearman and Kärber equation.

One-step growth curves. We incubated each cell line with CVA21 at a MOI of 3.0 for $2 \mathrm{~h}$ at $37^{\circ} \mathrm{C}$. After this incubation, we centrifuged the cells and removed unincorporated virus. We resuspended the cells in fresh growth medium at predetermined time points $(2,4,6,18,12$ and 24 hours), collected the cell 
pellets and froze them at $-80{ }^{\circ} \mathrm{C}$. After the completion of all time points, we thawed all of the samples and cleared the cellular debris by centrifugation, yielding a cleared cell lysate fraction.

MiRNA mimics. We purchased miRNA mimics from Dharmacon. The control miRNA mimic corresponded to a C. elegans miRNA with no predicted miRTs in mammalian cells, according to the manufacturer. We transfected the miRNA mimics with Mirus RNA transfection reagent at a $200 \mathrm{nM}$ concentration. Four hours after the mimic transfection, we infected the cells with WT, miRT or RevT CVA21 at an MOI of 1.0. Twenty-four hours after infection, we collected the cells for 3-(4,5-dimethylthiazol-2-yl)-2,5-diphenyltetrazolium bromide (MTT) viability assay and collected the supernatants for titration.

In vivo experiments. All in vivo experiments were approved by the Mayo Clinic Institutional Animal Care and Use Committee. We obtained CB17 ICR-SCID mice from Harlan. We irradiated the mice and implanted them with $5 \times 10^{6}$ Kas $6 / 1$ or Mel-624 cells in the right flank. When tumors reached an average size of $0.5 \mathrm{~cm} \times 0.5 \mathrm{~cm}$, we treated the tumors with $1 \times 10^{6}$ CVA21. We measured tumor volume with a hand-held caliper and collected blood by retroorbital bleeding. Histological and pathological analysis of mice was performed by Mayo Clinic Scottsdale Research Histology after terminal perfusion with $4 \%$ paraformaldehyde.

Statistical analyses. We used a two-tailed unpaired $t$-test to calculate statistical significance.

Note: Supplementary information is available on the Nature Medicine website.

\section{ACKNOWLEDGMENTS}

We thank D. Jelenik (Mayo Clinic) for Kas 6/1 multiple myeloma cells, R. Vile O (Mayo Clinic) for Mel-624 melanoma cells and Y. Ikeda (Mayo Clinic) for lentiviral transfer plasmids. We thank E. Rieder and E. Wimmer (State University of NewYork-Stony Brook) for the initial construction of an infectious CVA21 clone. Many thanks to A. Dufresne and M. Gromeier (Duke University) for kindly providing an optimized CVA21 clone. We also thank B. Brown and (. L. Naldini for helpful discussions.

\section{AUTHOR CONTRIBUTIONS}

E.J.K. and E.M.H. performed in vitro experiments. E.J.K., E.M.H. and S.G. performed in vivo experiments. E.J.K. and S.J.R. designed experiments and wrote the manuscript. S.J.R. supervised the project.

Published online at http://www.nature.com/naturemedicine/

Reprints and permissions information is available online at http://npg.nature.com/ Oo. reprintsandpermissions/

1. Zhao, Y. et al. Dysregulation of cardiogenesis, cardiac conduction, and cell cycle in mice lacking miRNA-1-2. Cell 129, 303-317 (2007).

2. Zhang, B., Pan, X., Cobb, G.P. \& Anderson, T.A. MicroRNAs as oncogenes and tumor suppressors. Dev. Biol. 302, 1-12 (2007).

3. van Rooij, E. et al. Control of stress-dependent cardiac growth and gene expression by a microRNA. Science 316, 575-579 (2007).

4. Lagos-Quintana, M. et al. Identification of tissue-specific microRNAs from mouse. Curr. Biol. 12, 735-739 (2002).

5. Bartel, D.P. MicroRNAs: genomics, biogenesis, mechanism, and function. Cell 116, 281-297 (2004).

6. Kelly, E. \& Russell, S.J. History of oncolytic viruses: genesis to genetic engineering. Mol. Ther. 15, 651-659 (2007).
7. Aghi, M. \& Martuza, R.L. Oncolytic viral therapies-the clinical experience. Oncogene 24, 7802-7816 (2005).

8. Thorne, S.H., Hermiston, T. \& Kirn, D. Oncolytic virotherapy: approaches to tumor targeting and enhancing antitumor effects. Semin. Oncol. 32, 537-548 (2005).

9. Brown, B.D., Venneri, M.A., Zingale, A., Sergi Sergi, L. \& Naldini, L. Endogenous microRNA regulation suppresses transgene expression in hematopoietic lineages and enables stable gene transfer. Nat. Med. 12, 585-591 (2006).

10. Gottwein, E., Cai, X. \& Cullen, B.R. A novel assay for viral microRNA function identifies a single nucleotide polymorphism that affects Drosha processing. J. Virol. 80, 5321-5326 (2006)

11. Schiff, G.M. \& Sherwood, J.R. Clinical activity of pleconaril in an experimentally induced coxsackievirus A21 respiratory infection. J. Infect. Dis. 181, 20-26 (2000).

12. Dekel, B., Yoeli, R., Shulman, L., Padeh, S. \& Passwell, J.H. Localized thigh swelling mimicking a neoplastic process: involvement of coxsackie virus type A21. Acta Paediatr. 91, 357-359 (2002).

13. Knipe, D.M. et al. Fields Virology, 5th Edition. Enteroviruses: Polioviruses, Coxsack ieviruses, Echoviruses, and Newer Enteroviruses Ch. 25 (Lippincott, Williams and Wilkins, Philadelphia, 2007).

14. Skelding, K.A., Barry, R.D. \& Shafren, D.R. Systemic targeting of metastatic human breast tumor xenografts by Coxsackievirus A21. Breast Cancer Res. Treat. published online, doi:10.1007/s10549-008-9899-2 (7 February 2008).

15. Au, G.G., Lindberg, A.M., Barry, R.D. \& Shafren, D.R. Oncolysis of vascular malignant human melanoma tumors by Coxsackievirus A21. Int. J. Oncol. 26, 1471-1476 (2005).

16. Baskerville, S. \& Bartel, D.P. Microarray profiling of microRNAs reveals frequent coexpression with neighboring miRNAs and host genes. RNA 11, 241-247 (2005).

17. Brown, B.D. et al. Endogenous microRNA can be broadly exploited to regulate transgene expression according to tissue, lineage and differentiation state. Nat. Biotechnol. 25, 1457-1467 (2007).

18. Nelson, P.T. et al. Microarray-based, high-throughput gene expression profiling of microRNAs. Nat. Methods 1, 155-161 (2004).

19. Rao, P.K., Kumar, R.M., Farkhondeh, M., Baskerville, S. \& Lodish, H.F. Myogenic factors that regulate expression of muscle-specific microRNAs. Proc. Natl. Acad. Sci. USA 103, 8721-8726 (2006).

20. Rohll, J.B., Moon, D.H., Evans, D.J. \& Almond, J.W. The $3^{\prime}$ untranslated region of picornavirus RNA: features required for efficient genome replication. J. Virol. 69, 7835-7844 (1995)

21. Dufresne, A.T. \& Gromeier, M. A nonpolio enterovirus with respiratory tropism causes poliomyelitis in intercellular adhesion molecule 1 transgenic mice. Proc. Natl. Acad. Sci. USA 101, 13636-13641 (2004).

22. Domingo, E., Menendez-Arias, L. \& Holland, J.J. RNA virus fitness. Rev. Med. Virol. 7, 87-96 (1997).

23. Alonso, M.M. et al. Combination of the oncolytic adenovirus ICOVIR-5 with chemotherapy provides enhanced anti-glioma effect in vivo. Cancer Gene Ther. 14, 756-761 (2007).

24. Hingorani, M. et al. Combining radiation and cancer gene therapy: a potential marriage of physical and biological targeting? Curr. Cancer Drug Targets 7, 389-409 (2007).

25. Qiao, J. et al. Purging metastases in lymphoid organs using a combination of antigennonspecific adoptive T cell therapy, oncolytic virotherapy and immunotherapy. Nat. Med. 14, 37-44 (2008).

26. Barber, G.N. Vesicular stomatitis virus as an oncolytic vector. Viral Immunol. 17, 516-527 (2004).

27. Qiao, J. et al. Cyclophosphamide facilitates antitumor efficacy against subcutaneous tumors following intravenous delivery of reovirus. Clin. Cancer Res. 14, 259-269 (2008)

28. Heise, C.C., Williams, A.M., Xue, S., Propst, M. \& Kirn, D.H. Intravenous administration of ONYX-015, a selectively replicating adenovirus, induces antitumoral efficacy. Cancer Res. 59, 2623-2628 (1999).

29. Roberts, L. Infectious disease. Vaccine-related polio outbreak in Nigeria raises concerns. Science 317, 1842 (2007).

30. Grimm, D. \& Kay, M.A. Combinatorial RNAi: a winning strategy for the race against evolving targets? Mol. Ther. 15, 878-888 (2007)

31. Parato, K.A., Senger, D., Forsyth, P.A. \& Bell, J.C. Recent progress in the battle between oncolytic viruses and tumours. Nat. Rev. Cancer 5, 965-976 (2005). 